\title{
Adolescent Sports Behavior and Social Networks: The Role of Social Efficacy and Self-Presentation in Sports Behavior
}

\author{
Lei Lei, ${ }^{1,2}$ Huifang Zhang $\mathbb{D}^{2}{ }^{2}$ and Xin Wang ${ }^{3}$ \\ ${ }^{1}$ Sports Department, Northwest A\&F University, Yangling 712100, China \\ ${ }^{2}$ School of Economics and Finance, Xi'an Jiaotong University, Xi'an 710064, China \\ ${ }^{3}$ Development Planning Office, Beijing Institute of Computer Technology and Application, Beijing 100854, China \\ Correspondence should be addressed to Huifang Zhang; zhanghuifang@xjtu.edu.cn
}

Received 18 June 2020; Revised 16 July 2020; Accepted 21 July 2020; Published 29 August 2020

Guest Editor: Liang Wang

Copyright $\odot 2020$ Lei Lei et al. This is an open access article distributed under the Creative Commons Attribution License, which permits unrestricted use, distribution, and reproduction in any medium, provided the original work is properly cited.

\begin{abstract}
Social networks are a complex system that members communicate, create new connections or destroy existing connections, and further deliver major impacts on each member's life. Given the spread of the Internet and increased academic pressure, sedentary and prescreen behaviors are very common among adolescents; meanwhile, sports behaviors are gradually decreasing. This situation has had an adverse effect on health. This paper used a questionnaire survey to investigate the influence of social networks on adolescent sports behavior, including the intermediary role of social efficacy and moderating effect of self-presentation. The questionnaire survey was conducted on 568 students from 6 high schools in Shaanxi, Henan, and Shandong Provinces. After this, factor analysis and weighted least squares method were used for the empirical test. Based on theoretical and empirical analysis, this paper found the following: (1) Social networks of adolescents have obvious positive predictive effects on their sports behavior. A single online social network and an offline social network, instrumental network, emotional network, and mixed network have obvious positive predictive effects on adolescent sports behaviors. However, under the influence of multiple types of social networks, an offline social network has a negative predictive effect, while a mixed network has effects that are not as obvious. (2) Social efficacy plays an intermediary role in the relationship between social network and adolescent sports behavior. (3) The moderating effect of self-presentation is not significant.
\end{abstract}

\section{Introduction}

Today, nearly four-fifths of the world's adolescents lack enough physical activity, and the situation for Chinese adolescents is even worse. Sedentary behavior and prescreen behavior are very common in adolescents' study and daily life activities. In 2019, the overweight and obesity rates of Chinese young students were $28.5 \%$ and $11.7 \%$, respectively. The myopia or short sight rate for primary school students, middle school students, and college students is also increasing, currently ranging from $45.7 \%$ to $87.7 \%$. Adolescents are the future of the world, and they are a group that needs special attention. Active participation in physical activities is conducive to the development of both the physical and mental health of adolescents, and it is thus an urgent task to promote greater adolescent physical activity levels.
In view of the basic trend of social development, which is changing from relatively closed small group to increasingly open networked community life, human society has reached an unprecedented level in terms of network structure, network connection density, and network member communication. Social networking not only is a static relationship structure but also offers a variety of dynamics for the now microprocess of cognition and interpersonal interaction. To pay close attention to the complex, unique, and continuous interaction between social network and actors, it is necessary to discuss the sociability of actors and their embedded network relationships. Although the influence and intervention of social networking on health risk behaviors, such as smoking, alcohol abuse, and sexual behavior of adolescents, have been extensively studied, the changes and ongoing development of adolescents' physical activity 
behavior, a complex social problem, need to be further studied at the level of social networking for the most relevant factors, theories, and mechanisms.

Compared to early childhood and adulthood, adolescents are more influenced by their peers during their process of socialization $[1,2]$. Studies have found that social networking can not only promote individuals' participation in collective actions and "catalyze" large-scale collective actions but also establish cross-organizational connections through its networks [3]. Making friendship with others can increase adolescents' motivation for sports. For example, distance cycling in front of friends or in a group will be greater in length than when they are cycling alone, indicating that one's peers do enhance the enthusiasm for sports activities [4]. Indeed, if adolescents have more friends and a larger proportion of same-sex friends, they will participate in more physical activities after school. The intensity of physical activity during recess also positively correlates with the number of friends who participate in sports. This situation also varies according to gender; for example, boys are more easily affected by their network of friends than girls are [5]. In addition, researchers believe that adolescents' physical activity behavior is influenced by their siblings and friends. Friends have a greater influence on adolescent participation in organized physical activities, while siblings mainly affect informal and spontaneous physical activity participation. Besides, the supports of teachers also influence adolescents for teachers and adolescents spending most time together in studying. Sometimes, the teacher-students relationship is important in adolescents' behavior because of regulations and commands. At the same time, parental support is crucial because it can reduce the internal perception barriers of adolescents and affect their daily participation in physical activities. If parents were and are also active participants in physical activity, their adolescent children will be more likely to show active physical activity behavior as well [6].

Although the social network environment should be considered for any intervention of adolescents' physical activities, it is worth noting that the influence of friends and partners on adolescents' physical activities is not regulated by their knowledge of those physical activities, but instead by the interdependent relationship between the network selected by the peers and their physical activity [7]. Therefore, in any intervention of physical activity behaviors of adolescents, one's choice of friends may be critical for promoting and maintaining health and positive behaviors. In other words, social networking may have a positive influence on adolescent sports behavior.

Given the popularization of computers, network platforms have gradually become the main channel for adolescents to use to reach out and make friends. Adolescents not only form offline social network but also form online social networks. Adolescents who are interested in physical activities online prefer to be closer to relevant topics and are more likely to provide information about their physical activities [8]. That is to say, given a digital background, not only may the offline social network actively promote adolescent sports behavior, but it also may promote individual sports behavior as well.
In reality, the theoretical model of "two paths and three layers" of individual behavior suggests that stable personality traits and external situations affect individual behaviors via internal cognitive processes. Moreover, social cognitive theory points out that individual behavior, subject cognition, and environment interact dynamically, while individual cognition is the core of such interaction [9]. Both theories demonstrate that internal cognition plays an important intermediary role between external situations and personal behavior. Bandura pointed out that a sense of efficacy is an important cognitive factor that not only affects an individual's choice of behavior but also determines the effort level and the ability to overcome obstacles [10].

Since self-efficacy is domain-specific, Fan and Mak put forward social efficacy [11] to show that there is an obvious positive correlation between social relations and social efficacy [12]. Individuals with low social efficacy tend to interpret uncertain social situations as dangerous and have negative reactions, including excessive self-concern, anxiety, and operational behavior, all obstacles used to avoid and withdraw from real social situations [13] and further reduce individual behaviors. To sum up then, in a real-life communication situation, social networks, social efficacy, and individual behavior are closely related. Indeed, social efficacy may play an intermediary role in the social network role in influencing adolescent sports behavior.

The popularity of computers and the diversification of network platforms now enable adolescents to present themselves at any time and share and learn on network platforms, such as Facebook or WeChat. With the rise of sports apps and quantitative equipment, many adolescents now use sports software to exercise and show and share themselves on social platforms. For example, the development of Keep and other fitness apps allows adolescents to not only learn the existing sports videos but also share their sports videos and sports records on the same platform and promote their continuous sports activity. On the one hand, this development may raise adolescents' attention toward their own health and strengthen their sports behavior; on the other hand, it can also make other adolescents undertake sports through the communication and sharing of them on social networks. Thus, the adolescents' selfpresentation on network platforms is likely to strengthen the influence of social networking on adolescent sports behavior. These above thoughts mean that diverse networks may promote adolescents' self-presentation and that self-presentation may encourage adolescents to do sports more frequently, which shows that self-presentation indeed has a moderating effect.

Through undertaking the above analysis, this paper examines the influence of social networking on adolescent sports behavior, the mediating effect of adolescents' social efficacy, and the moderating effect of self-presentation. Based on social cognition theory, this paper proposes a model that includes a mediating effect and a moderating effect and puts forward three hypotheses: H1: social networks positively promote adolescent sports behavior; H2: social efficacy plays a mediating role in the relationship between social network and adolescent sports behavior; and H3: self-presentation plays a moderating role for social networking and adolescent sports behavior. 
At the same time, to deeply analyze the influence of different social networks on adolescents sports, this study divides social networks into online and offline social platforms and emotional, instrumental, and mixed social networks. Of these, teachers and counselors belong to the instrumental network, family members and lovers belong to the emotional network, and roommates and classmates belong to a mixed network [11]. Through an analysis of social networks, social efficacy, and self-presentation, this paper offers both a theoretical basis and practical guidance for promoting the establishment of social networks and facilitating adolescent sports behavior.

\section{Materials and Methodology}

2.1. Subjects. The subjects were 630 adolescents from three junior middle schools and three senior high schools in Shaanxi, Henan, and Shandong Provinces. A total of 630 questionnaires were distributed, and of these, 568 valid questionnaires were collected, providing an effective recovery rate of $90.1 \%$. There were 296 boys ( $52.11 \%$ ) and 272 girls (47.89\%), 376 senior students, and 192 junior students. The average age of the subjects was 14.52 years $(\mathrm{SD}=1.68$, with the age range being 12-18).

2.2. Measurements. An adolescent sports behavior scale is based on the questionnaire by Mao et al. [14], which included 6 items (e.g., I usually take exercise with friends; I have the habit of exercising), and the questionnaire was scored using 5 answers (1 "totally inconsistent" to 5 "completely consistent"). In this study, the confirmatory factor analysis of the questionnaire fits well: $\chi^{2} / \mathrm{d} f=2.84$, RMSEA $=0.05, \mathrm{NFI}=0.995, \mathrm{GFI}=0.998$, and CFI $=0.997$. The internal concordance coefficient $\boldsymbol{\alpha}$ was 0.687 .

The social network scale uses a questionnaire designed by Park et al. [15], which contains 14 items and is divided into either two dimensions as (1) an online social network (6 items, e.g., Many of my friends in real life love sports) and (2) an offline social network (4 items, e.g., The number of communities I've join online), or three dimensions as (1) an instrumental social network (4 items, e.g., I have a close relationship with my class teacher), (2) an emotional network (4 items, e.g., I have a close relationship with my relatives of the same age), and (3) a mixed network (4 items, e.g., I have a close relationship with my friends in real life).

Some of the items in the questionnaire on different dimensions were the same. For example, "I have a close relationship with my friends in real life" is a measurement item of both an offline social network and a mixed network. The questionnaire is scored using 5 points ( 1 "totally inconsistent" to 5 "completely consistent"). In the current study, the confirmatory factor analysis of the questionnaire fits well: $\chi^{2} / \mathrm{d} f=2.84$, RMSEA $=0.06$, NFI $=0.998$, GFI $=0.999$, and $\mathrm{CFI}=0.999$. The internal concordance coefficient $\boldsymbol{\alpha}$ was 0.654 . The coefficients $\boldsymbol{\alpha}$ of each dimension were 0.940 (online social network), 0.936 (offline social network), 0.898 (instrumental social network), 0.766 (emotional social network), and 0.847 (mixed social network).
The social efficacy scale used a questionnaire compiled by Jeong and Kim [16]. There were 6 items on this questionnaire in the study (e.g., online communication, I can easily become friends with other people; I can easily talk to unfamiliar people). The questionnaire was scored using 5 points (1 "totally inconsistent "to 5 " completely consistent"). In this study, the confirmatory factor analysis of the questionnaire fits well: $\chi^{2} / \mathrm{d} f=1.24$, RMSEA $=0.02, \mathrm{NFI}=0.998$, $\mathrm{GFI}=0.999$, and $\mathrm{CFI}=0.999$. The internal concordance coefficient $\alpha$ was 0.634 .

The self-presentation scale used a questionnaire compiled by Kim and Lee [17]. It included 4 items (e.g., I will post photos that show the real me; I don't mind sharing some bad things that happened to me on the Internet). The questionnaire was scored based on 5 points ( 1 "totally inconsistent" to 5 "completely consistent"). In this study, the confirmatory factor analysis of the questionnaire fits well: $\chi^{2} / \mathrm{d} f=2.24$, RMSEA $=0.02$, NFI $=0.998$, GFI $=0.999$, and $\mathrm{CFI}=0.999$. The internal concordance coefficient $\alpha$ was 0.820 .

2.3. Programs and Data Processing. In this current study, after obtaining the consent of the school leaders, teachers, and students, the questionnaire practice was explained by highly trained surveyors in accordance with standardized instruction, and all questionnaires were collected immediately. SPSS 22.0 software was used to analyze the data, and PROCESS V3.0 was used to test the mediating effect.

First, we used the factor analysis method to reduce the dimensionality of variables. The mathematical model was

$$
\left\{\begin{array}{l}
x_{1}=a_{11} f_{1}+a_{12} f_{2}+a_{13} f_{3}+\cdots+a_{1 k} f_{k}+\varepsilon_{1}, \\
x_{2}=a_{21} f_{1}+a_{22} f_{2}+a_{23} f_{3}+\cdots+a_{2 k} f_{k}+\varepsilon_{2}, \\
x_{3}=a_{31} f_{1}+a_{32} f_{2}+a_{33} f_{3}+\cdots+a_{3 k} f_{k}+\varepsilon_{3}, \\
\cdots \\
x_{p}=a_{p 1} f_{1}+a_{p 2} f_{2}+a_{p 3} f_{3}+\cdots+a_{p k} f_{k}+\varepsilon_{p} .
\end{array}\right.
$$

For equation (1), $x_{i}$ is the standardized variable; $f_{i}$ is the factor variable; $\varepsilon$ is the special factor; $a_{i j}$ is the factor load; $k<p$. A matrix was used to simplify equation (1) as follows:

$$
\mathbf{X}=\mathbf{A F}+\varepsilon,
$$

where $\mathbf{F}$ is the factor variable matrix and $\mathbf{A}$ is a factor load matrix and satisfies $\operatorname{cov}(F, \varepsilon)=0, D(F)=I_{m}, D(\varepsilon)=\delta_{i}$.

The factor load $a_{i j}$ represents the degree of correlation between $X_{i}$ and $F_{i}$ and the square sum of the elements in the $i$-th row of the factor load matrix where $\mathbf{A}$ is $h_{i}^{2}=\sum_{j=1}^{k} a_{i j}^{2}$, which represents the explanatory power of all the factor variables for the total variance of $X_{i}$, and $S_{j}=\sum_{i=1}^{p} a_{i j}^{2}$ is the variance contribution of the variable $F_{j}$.

After determining the factor variables, the factor load matrix needed to be estimated. If we set the eigenvalues of the sample covariance matrix and the corresponding standard orthogonalized eigenvectors, as $\lambda_{1} \geq \lambda_{2} \geq \cdots \geq \lambda_{p} \geq 0$, $e_{1}, e_{2}, \ldots, e_{p}$, then the covariance matrix can be decomposed into 


$$
\sum=\mathbf{U}\left(\begin{array}{cccc}
\lambda_{1} & & & 0 \\
& \lambda_{2} & & \\
& & \ldots & \\
& & & \lambda_{p}
\end{array}\right) \mathbf{U}^{\prime}=\sum_{i=1}^{p} \lambda_{i} e_{i} e_{i}^{\prime} .
$$

When the last several eigenvalues are small, the covariance matrix can be approximately decomposed into

$$
\begin{aligned}
\sum & \approx\left(\sqrt{\lambda_{1}} e_{1}, \ldots, \sqrt{\lambda_{m}} e_{m}\right)\left[\begin{array}{c}
\sqrt{\lambda_{1}} e_{1}^{\prime} \\
\cdots \\
\sqrt{\lambda_{m}} e_{m}^{\prime}
\end{array}\right]+\left(\begin{array}{ccc}
\sigma_{1}^{2} & & \\
& \sigma_{2}^{2} & \\
& & \\
& & \\
& & \sigma_{p}^{2}
\end{array}\right) \\
& =\mathbf{A A}^{\prime}+\sum_{\varepsilon},
\end{aligned}
$$

where $\mathbf{A}$ is the factor covariance matrix. Since the factor loading matrix is not unique, factor rotation was executed to make the meaning of the common factor clearer. Considering the orthogonal rotation of two factors, the orthogonal matrix then became

$$
\mathbf{Q}=\left(\begin{array}{cc}
\cos \phi & -\sin \phi \\
\sin \phi & \cos \phi
\end{array}\right),
$$

and set

$$
\mathbf{B}=\mathbf{A} \mathbf{Q}=\left(b_{i j}\right), \quad i=1,2, \ldots, p, j=1,2,
$$

where $\mathbf{B}$ is the rotation factor load matrix. At this time, we required the variance of the two columns' data in $\mathbf{B}$ to be as large as possible, which also meant that the relative variance $V_{i}$ should also be as large as possible:

$$
V_{j}=\frac{1}{p} \sum_{i=1}^{p}\left(\frac{b_{i j}^{2}}{h_{i}^{2}}\right)^{2}-\sum_{i=1}^{p}\left(\frac{b_{i j}^{2}}{h_{i}^{2}}\right)^{2}, \quad j=1,2 .
$$

Making $\mathrm{d} V / \mathrm{d} \phi=0$, then $\phi$ should satisfy the following equation:

$$
\tan 4 \phi=\frac{D_{0}-\left(\left(2 A_{0} B_{0}\right) / p\right)}{C_{0}-\left(\left(A_{0}^{2}-B_{0}^{2}\right) / p\right)}
$$

where

$$
\begin{cases}A_{0}=\sum_{i=1}^{p} u_{i}, & B_{0}=\sum_{i=1}^{p} v_{i}, \\ C_{0}=\sum_{i=1}^{p}\left(u_{i}^{2}-v_{i}^{2}\right), & D_{0}=2 \sum_{i=1}^{p} u_{i} v_{i} \\ u_{i}=\left(\frac{a_{i 1}^{2}}{h_{i}}\right)^{2}-\left(\frac{a_{i i}^{2}}{h_{i}}\right)^{2}, & v_{i}=\frac{2 a_{i 1} a_{i 2}}{h_{i}^{2}} .\end{cases}
$$

Using SPSS for the factor analysis, taking adolescent sports and social network as examples, the scree plots were obtained. It can be seen from Figure 1 that the adolescent sports scale can extract one factor, while the social network scale can extract two factors.

\section{Results and Discussion}

3.1. Descriptive Analysis. The mean, standard deviation, and the correlation coefficient of each variable are shown in Table 1. These results show that there are obvious correlations between adolescent sports behavior and different social networks and also obvious correlations between social efficacy, self-presentation, social networking, and sports. In addition, social networks, especially offline social networks, have significant age differences. Different types of social networks and self-presentations also have gender differences. In order to explore the independent effects of social network on adolescent sports, age and gender were used here as the control variables.

Furthermore, we use histogram to basically analyze the sample. From Figure 2(a), it can be seen that the sample is balanced and distributed between male and female, indicating the sample can reflect reality well. In different gender group (Figure 2(b)), the number of adolescents who are 1314 years old is the most, while the number of 12 years old adolescents is the least.

From Figure 2(c), during the group of 12 years old adolescents, the scores of five social networks are all high, indicating that, for 12-year-olds, their social network type, density, and intensity are stronger. For 13-14 years old adolescents, all types of social networks are not high, especially online social network. Its average value is 1.2 which means the online social network is not strong. This may because the academic pressure for adolescents in the stage is higher than other groups. For 15-16 years old adolescents, mixed social network and emotional social network are stronger than other social networks, and for 17-18 years old adolescents, online social network, mixed social network, and emotional social network are stronger than other two social networks. From Figure 2(d), no matter how old adolescents are, their sports activities are frequent. For example, for 13-14 years old adolescents, most adolescents do sports more than once a week; only $5 \%$ of adolescents do sports once a week or even less than once a week. From Figure 2, we can clearly know the distribution of sample, the social networks of different group, and the sports behavior of different groups; it helps to lay the foundation for the following empirical research.

\subsection{Results}

3.2.1. Influence of Social Networking on Adolescent Sports Behavior. Econometric models are constructed to test the relationships between different social networks and adolescent sports behavior:

$$
\text { Sport }_{i}=\beta X_{i}+\mathbf{P Q}+e_{1} .
$$

Among them, Sport is adolescent sports behavior, $X$ is an adolescents' social network that includes an online/offline social network and an instrumental/emotional/mixed social 


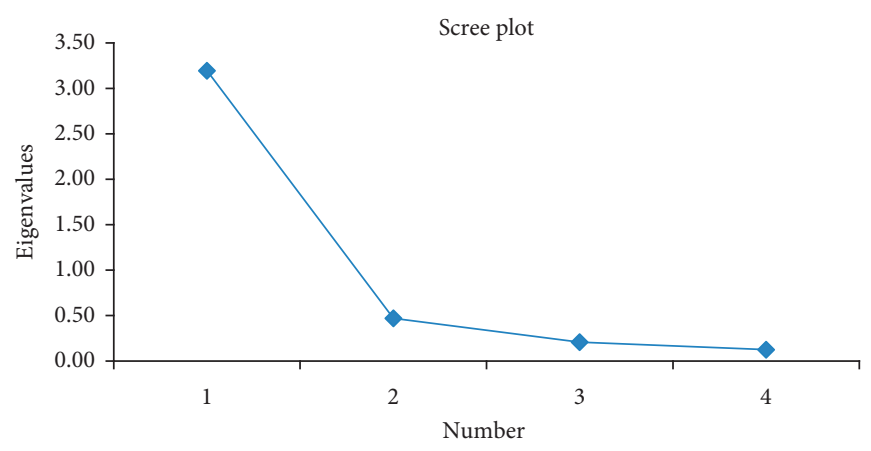

(a)

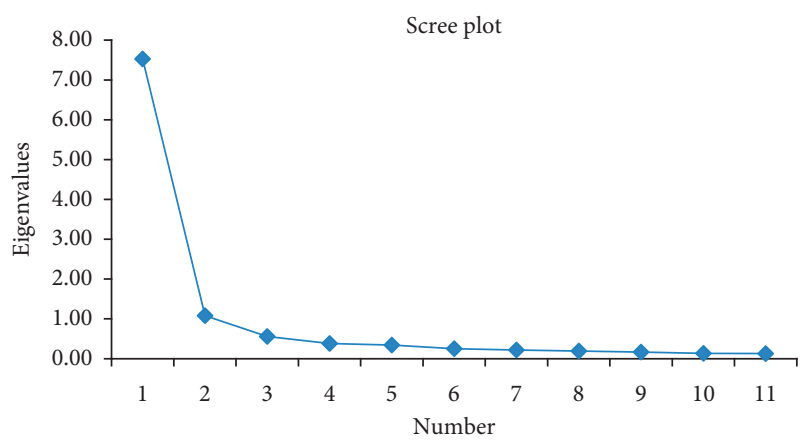

(b)

FIGURE 1: Scree plot of adolescent sports (a) and social network (b).

TABLE 1: Mean, standard deviation, and correlation coefficient of variables.

\begin{tabular}{|c|c|c|c|c|c|c|c|c|c|c|c|c|c|}
\hline & $\mathrm{M}$ & $\mathrm{SD}$ & 1 & 2 & 3 & 4 & 5 & 6 & 7 & 8 & 9 & 10 & 11 \\
\hline 1. Age & 14.53 & 1.69 & 1 & & & & & & & & & & \\
\hline 2. Gender & 0.53 & 0.50 & -0.018 & 1 & & & & & & & & & \\
\hline 3. Sports & 2.88 & 0.93 & -0.042 & -0.076 & 1 & & & & & & & & \\
\hline 4. Social network & 2.08 & 0.87 & $-0.004^{*}$ & $-0.105^{*}$ & $0.858^{*}$ & 1 & & & & & & & \\
\hline 5. Online social network & 2.09 & 0.89 & $0.015^{*}$ & -0.084 & $0.744^{* *}$ & $0.967^{* *}$ & 1 & & & & & & \\
\hline 6. Offline social network & 2.36 & 0.64 & -0.015 & $-0.113^{*}$ & $0.903^{* *}$ & $0.988^{* *}$ & $0.916^{* *}$ & 1 & & & & & \\
\hline 7. Mixes social network & 2.30 & 0.71 & -0.018 & $-0.126^{* *}$ & $0.876^{* *}$ & $0.945^{*}$ & $0.863^{* *}$ & $0.966^{* *}$ & 1 & & & & \\
\hline $\begin{array}{l}\text { 8. Instrumental social } \\
\text { network }\end{array}$ & 2.34 & 0.66 & 0.000 & -0 . & $0.734^{* *}$ & $0.944^{* *}$ & $0.923^{* *}$ & $0.924^{* *}$ & $0.823^{* *}$ & 1 & & & \\
\hline $\begin{array}{l}\text { 9. Emotional social } \\
\text { network }\end{array}$ & 14.53 & 1.69 & -0.033 & -0.066 & $0.965^{* *}$ & $0.844^{* *}$ & $0.724^{* *}$ & $0.891^{* *}$ & $0.850^{* *}$ & $0.711^{* *}$ & 1 & & \\
\hline 10. Social efficacy & 2.41 & 0.71 & -0.058 & -0.078 & $0.980^{* *}$ & $0.838^{* *}$ & $0.725^{* *}$ & $0.884^{* *}$ & $0.880^{* *}$ & $0.712^{* *}$ & $0.916^{* *}$ & 1 & \\
\hline 11. Self-presentation & 2.43 & 0.96 & 0.006 & $-0.116^{* *}$ & $0.808^{* *}$ & $0.810^{* *}$ & $0.710^{* *}$ & $0.848^{* *}$ & $0.895^{* *}$ & $0.662^{* *}$ & $0.816^{* *}$ & $0.780^{* *}$ & 1 \\
\hline
\end{tabular}

Note: $N=568$, gender is a dummy variable, female $=0$, and male $=1 .{ }^{*} p<0.05 ;^{* *} p<0.01$.

network, $\mathbf{Q}$ is the control variable matrix, and $\boldsymbol{e}_{1}$ is the regression residual. The empirical results are shown in Table 2.

The results in Table 2 indicate that adolescents' social networks have a significant positive effect on their sports behavior $(\beta=0.860, p<0.001)$. The wider the homogeneity of these adolescents' social relationship is, the more frequent their sports behavior becomes. Online social networks and offline social networks have positive effects on adolescent sports behavior, respectively. However, when adolescents have both an offline social network and an online social network, the offline social network has a positive effect on adolescent sports $(\beta=1.381, p<0.001)$, while the online social network has a negative effect on adolescent sports $(\beta=0.517, p<0.001)$. An instrumental network, emotional network, and mixed network have positive effects on adolescent sports, respectively, with coefficients of $0.735,0.964,0.880$, and are significant at a $1 \%$ level. However, when adolescents have the three kinds of above social relations at the same time, the emotional network and the mixed network still have positive effects on adolescent sports, but the influence of the instrumental network on adolescent sports then becomes insignificant.
3.2.2. Mediating Effect of Social Efficacy. According to Wen and Ye [18], it is necessary to test the parameters of three regression equations to verify the mediating effect:

$$
\begin{aligned}
\text { Sport }_{i} & =c \mathrm{SN}_{i}+\mathbf{P Q}+e_{2}, \\
\mathrm{SE}_{i} & =\alpha \mathrm{SN}_{i}+\mathbf{P Q}+e_{3}, \\
\mathrm{Sport}_{i} & =c^{\prime} \mathrm{SP}_{i}+b \mathrm{SE}_{i}+\mathbf{P Q}+e_{4} .
\end{aligned}
$$

Among these, $\mathrm{SN}$ is social network, $\mathrm{SE}$ is social efficacy, $\mathbf{Q}$ is the control variable matrix, and $e_{2} \sim e_{4}$ are the regression residuals. Further, $c$ is the total effect of the independent variable (social network) on the dependent variable (adolescent sports behavior); $a$ is the effect of social network on the intervening variable (social efficacy); $b$ is the effect of social efficacy on adolescent sports behavior after controlling for the influence of social network; $c^{\prime}$ is the direct effect of social network on adolescent sports behavior after controlling for the influence of social efficacy. The mediating effect is tested in five steps: first, test the coefficient $c$ of equation (11); if $c$ is significant, the intermediary effect is significant; otherwise, there is a masking effect. But whether it is significant or not, follow-up tests are carried out. 


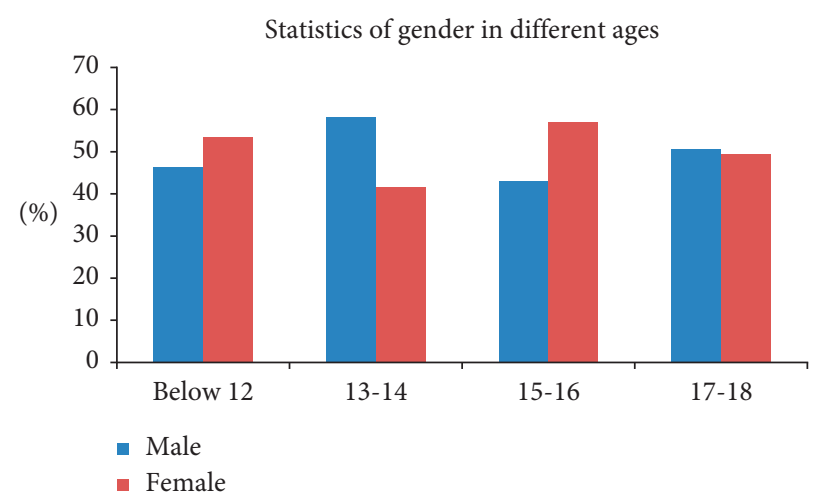

(a)

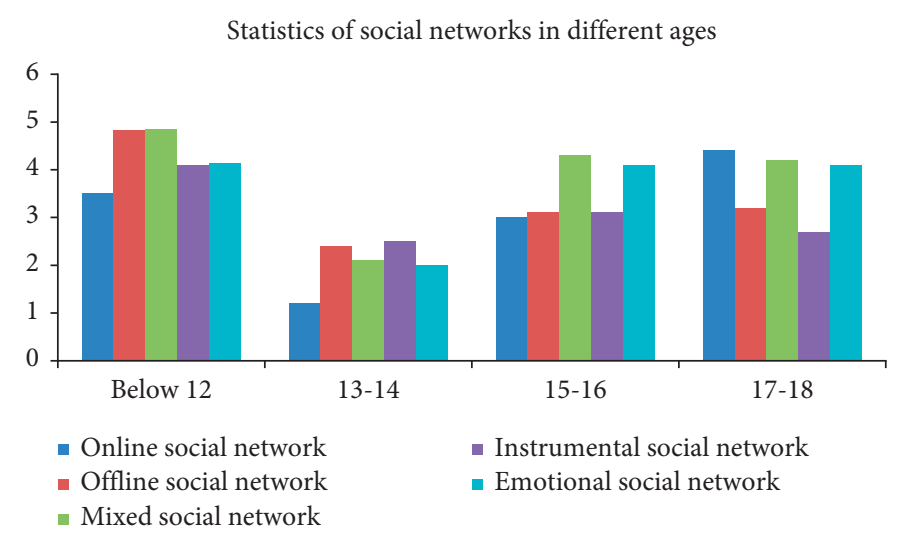

(c)

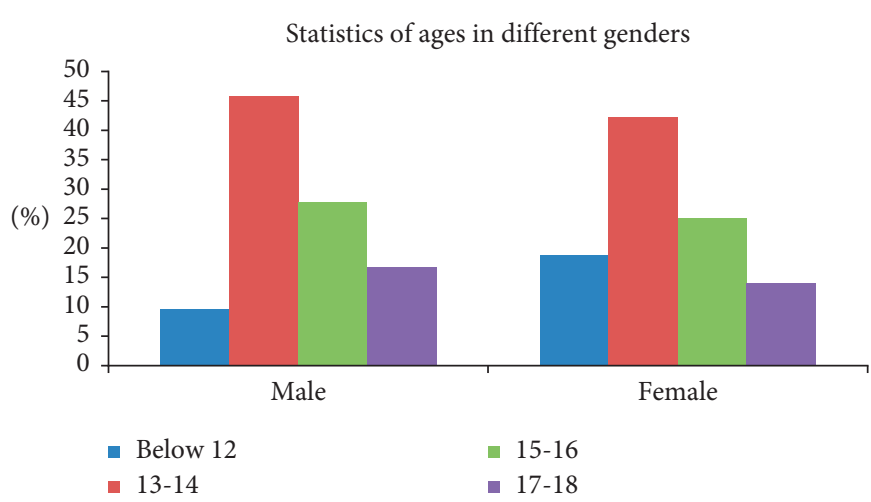

(b)

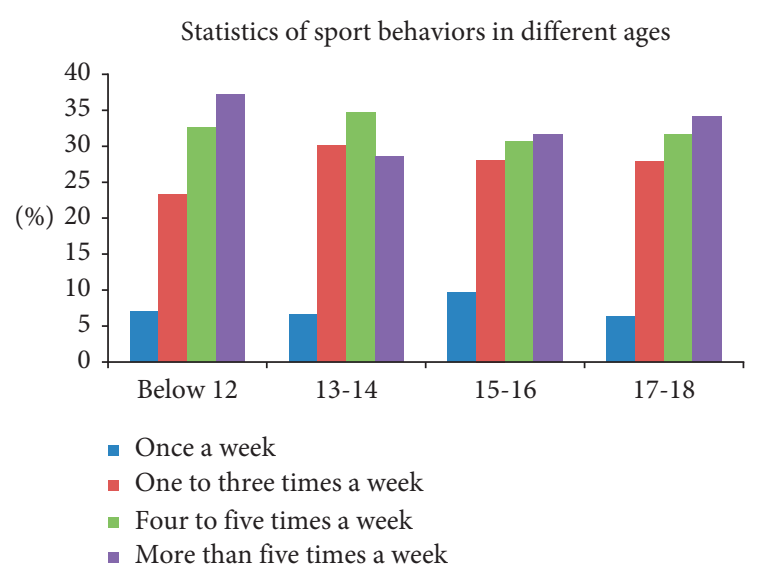

(d)

FIGURE 2: Statistic of adolescent social networks and sports behavior.

TABLE 2: Estimated results of the influence of social network on adolescent sports behavior.

\begin{tabular}{|c|c|c|c|c|c|c|c|c|}
\hline Variables & & & & Adolescent spo & rts behavior & & & \\
\hline Constant & $0.316(1.568)$ & $0.471^{*}(1.794)$ & $0.210(1.240)$ & $0.069(0.465)$ & $0.357(1.336)$ & $0.100(0.966)$ & $0.179(0.945)$ & $0.102(1.071)$ \\
\hline Age & $-0.023^{*}(-1.667)$ & $-0.031^{*}(-1.767)$ & $-0.016(-1.427)$ & $-0.007(-0.737)$ & $-0.025(-1.366)$ & $-0.006(-0.859)$ & $-0.015(-1.157)$ & $-0.007(-1.113)$ \\
\hline Gender & $0.028(0.599)$ & $-0.028(-0.458)$ & $0.053(1.356)$ & $0.074^{* *}(2.154)$ & $0.004(0.068)$ & $-0.024(-1.004)$ & $0.070(1.596)$ & $0.004(0.203)$ \\
\hline $\begin{array}{l}\text { Social } \\
\text { network }\end{array}$ & $0.860^{* * *}(37.052)$ & - & - & - & - & - & - & - \\
\hline $\begin{array}{l}\text { Online social } \\
\text { network }\end{array}$ & - & $0.744^{* * *}(24.673)$ & - & $-0.517^{* * *}(-12.194)$ & - & - & - & - \\
\hline $\begin{array}{l}\text { Offline social } \\
\text { network }\end{array}$ & - & - & $0.905^{* * *}(45.545)$ & $1.381^{* * *}(32.455)$ & - & - & - & - \\
\hline $\begin{array}{l}\text { Instrumental } \\
\text { network }\end{array}$ & - & - & - & - & $0.735^{* * *}(23.892)$ & - & - & $0.016(0.818)$ \\
\hline $\begin{array}{l}\text { Emotional } \\
\text { network }\end{array}$ & - & - & - & - & - & $0.964^{* * *}(81.163)$ & - & $0.793^{* * *}(38.244)$ \\
\hline $\begin{array}{l}\text { Mixed } \\
\text { network }\end{array}$ & - & - & - & - & - & - & $0.880^{* * *}(40.217)$ & $0.189^{* * *}(7.352)$ \\
\hline Adj- $R^{2}$ & 0.737 & 0.554 & 0.816 & 0.858 & 0.538 & 0.931 & 0.767 & 0.942 \\
\hline
\end{tabular}

Note: $t$ value is in parentheses. Symbols ${ }^{*}, * *$, and ${ }^{* * *}$ indicate that coefficient is significant at $10 \%, 5 \%$, and $1 \%$ levels.

Second, test the coefficient $a$ of equation (12) and the coefficient $b$ of equation (13) in turn; if both are significant, then the indirect effect is significant; go to the fourth step; if at least one is not significant, go to the third step. Third, use Bootstrap method to directly check $H 0: a b=0$. If it is significant, then the indirect effect is significant; go to the fourth step; otherwise, the indirect effect is not significant; stop the analysis. Forth, examining the coefficient $c^{\prime}$ of equation (13), if it is not significant, the direct effect is not significant, indicating that there is only an intermediary effect. If it is significant, that is, the direct effect is significant, go to the fifth step. Fifth, compare the signs of $a b$ and $c^{\prime}$. If they are the same, it is partly intermediary effect. If the sign is different, it is a masking effect. If $a, b$ and $c$ are all significant, the mediation effect is significant; otherwise, there are other effects. For example, if $c$ is not significant, there is a masking effect; if $c^{\prime}$ is significant, there also exist direct effects. Besides the above five steps, a nonparametric percentile Bootstrap 
method was used to test the mediating effect. If the $95 \%$ confidence interval does not contain a zero, then the mediating effect becomes significant. The empirical regression results and significance test results for the mediating effect are shown in Table 3.

The results in Table 3 show that $R^{2}$ in the three equations was bigger than 0.7 and the goodness of fit for the model was good. In equation (11), social network has a significant positive effect on adolescent sports behavior, $c=0.860, p<0.001$. In equation (12), social network has a significant effect on social efficacy, $c=0.839, p<0.001$. In equation (13), social network and social efficacy have a significant effect on adolescent sports behavior, $c^{\prime}=0.122, b=0.879, p<0.001$. According to the mediating effect test procedure of Wen and Ye [18], the mediating effect of social efficacy is significant because $a, b, c$, and $c^{\prime}$ are all significant. Further, the Bootstrap method was used to test the mediating effect of social efficacy. 5000 Bootstrap samples were randomly selected from the original sample for indirect effect estimation. Table 3 shows the estimated value of the indirect effect of social efficacy to be 0.7374 and $95 \%$ confidence intervals were $[0.7007,0.7704]$, excluding zero. Thus, the mediating effect of social efficacy became significant.

3.2.3. Moderating Effect of Self-Presentation. As for the moderating effect of self-presentation, the following econometric model was constructed:

$$
\text { Sport }_{i}=\alpha \mathrm{SN}_{i}+\beta \mathrm{SP}_{i}+\gamma \mathrm{SN}_{i} * \mathrm{SP}_{i}+\mathrm{PQ}+e_{5},
$$

where SP is the self-presentation. The stepwise regression method was used to estimate the moderating effect of selfpresentation. The results are shown in Table 4.

The results of the study show that although both social networking and self-presentation have significant positive effects on adolescent sports behavior, the interaction terms of social network and self-presentation do not have any significant influence on adolescent sports behavior, thereby indicating that the moderating effect of self-presentation is not significant.

In addition, this study found that age has a negative predictive effect on adolescent sports behavior; that is, the older the adolescents are, the less likely they are to exercise. Gender had no significant effect on the prediction of adolescent sports behavior.

\subsection{Discussion}

3.3.1. Influence of Social Networks on Adolescent Sports Behavior. The results of this study indicate that social networks have significant influences on adolescent sports behavior, demonstrating that adolescents with larger and denser social networks have more frequent sports behavior. People are mutually linked to each other, and so are their sports behaviors. Thus, the existence of social networks means that persons' movements are interdependent [19]. Sports behavior can be spread from person to person, and there is also a phenomenon called "peer effects" [20], which is just as the proverb says: "If you live with a lame person you will learn to limp." However, it is interesting that, with the strengthening of the social network establishment, the effects of online social networks and offline social networks on adolescent sports behavior are not the same. When adolescents have both an offline social network and an online social network, the offline social network has a positive effect on adolescent sports, while the online social network has a negative effect on adolescent sports. This outcome may be because, at the present stage, Chinese adolescents make friends online mainly through games, chats, and other ways [21]. Moreover, adolescents in junior and senior high schools have heavy daily learning tasks and usually surf the Internet for leisure and entertainment. Therefore, most adolescents' online social network relationships are entertainment groups. Besides, during the formation of online network relationships, most adolescents will use computers or mobile phones for a long time, a factor that further reduces the possibility of adolescent sports [22]. Under the influence of the characteristics of network groups and the time used for online communication, offline social networks have a negative influence on adolescent sports behavior.

For social networks with different characteristics, the instrumental network, emotional network, and mixed network have positive effects on adolescent sports, respectively. In other words, without the influence of others, social relations, such as those with counselors, teachers, family members, and friends, have positive effects on adolescent sports behaviors. However, when adolescents have the above three kinds of social relations at the same time, the influence of the instrumental network, that is, counselors or teachers, on adolescent sports behavior is not significant. That is to say, the influence of counselors or teachers on adolescent sports behavior is easily affected by other people. This outcome may be because the instrumental network connectivity is not strong [23]. Even for teachers and students who live and study very closely, there is no similar influence like those of friends, indicating that although physical activity has the interpersonal infectivity of social network, its infectivity changes based on relative activities and the genders of friends [24]. For example, in a given social network, less active runners tend to affect more active runners, while the opposite is not true. Both men and women affect men, while only women affect other women [25]. The reason why it is easy for physical activity behaviors to spread through adolescent friendship or a family network is that first of all, adolescents are more willing to experience peer relations during adolescence, and their peer groups are based on common behaviors (sports, computer games, video games, diet, etc.). These directly or indirectly affect their physical activities and health [26]. Secondly, family relationship is the network relationship with the strongest connection among adolescents. Under the influence of that strong connection, an emotional network will have an obvious influence on adolescent sports behavior.

Overall, different types of social networks have positive effects on adolescent sports behavior separately, but different social networks are also interacted, so their influences on adolescent sports behavior will change by the interactions. 
TABLE 3: Estimated results and significance test results of mediating effect of social efficacy.

\begin{tabular}{|c|c|c|c|}
\hline Variables & $\begin{array}{l}\text { Equation (11) } \\
\text { Sports }\end{array}$ & $\begin{array}{l}\text { Equation (12) } \\
\text { Social efficacy }\end{array}$ & $\begin{array}{l}\text { Equation (13) } \\
\text { Sports }\end{array}$ \\
\hline Constant & $0.316(1.568)$ & $0.460^{* *}(2.148)$ & $-0.088(-1.191)$ \\
\hline Age & $-0.023^{*}(-1.667)$ & $-0.032^{* *}(-2.228)$ & $0.006(1.123)$ \\
\hline Gender & $0.028(0.599)$ & $0.018(0.364)$ & $0.012(0.716)$ \\
\hline Social network & $0.860^{* * *}(37.052)$ & $0.839^{* * *}(34.099)$ & $0.122^{* * *}(7.916)$ \\
\hline Social efficacy & - & - & $0.879^{* * *}(56.91)$ \\
\hline $\operatorname{Adj}-R^{2}$ & 0.737 & 0.706 & 0.956 \\
\hline \multicolumn{4}{|l|}{ Indirect effect(s) of SN on sport } \\
\hline Intervening variable: social contact & $\begin{array}{c}\text { Effect } \\
0.7374\end{array}$ & $\begin{array}{c}\text { Boot standard error } \\
0.0178\end{array}$ & $\begin{array}{c}\text { Boot LLCI } \\
0.7007\end{array}$ \\
\hline
\end{tabular}

Note: $t$ value is in parentheses. Symbols ${ }^{*},{ }^{* *}$, and ${ }^{* * *}$ indicate that coefficient is significant at $10 \%, 5 \%$, and $1 \%$ levels.

TABLE 4: Estimated results of moderating effect of self-presentation.

\begin{tabular}{lcrc}
\hline Variables & & Adolescent sports behavior \\
\hline Constant & $0.383^{*}(1.654)$ & $0.330^{*}(1.768)$ & $0.317^{*}(1.691)$ \\
Age & $-0.028^{*}(-1.764)$ & $-0.024^{*}(-1.936)$ & $-0.024^{*}(-1.916)$ \\
Gender & $0.036(0.673)$ & $0.049(1.134)$ & $0.046(1.069)$ \\
Self- presentation & $0.811^{* * *}(30.426)$ & $0.332^{* * *}(9.093)$ & $0.329^{* * *}(8.993)$ \\
Social network & - & $0.592^{* * *}(16.231)$ & $0.589^{* * *}(16.109)$ \\
Social network ${ }^{*}$ self-presentation & - & - & $0.014(0.891)$ \\
Adj- $R^{2}$ & 0.654 & 0.774 & 0.774 \\
\hline
\end{tabular}

Note: $t$ value is in parentheses. Symbols ${ }^{*},{ }^{* *}$, and ${ }^{* * *}$ indicate that coefficient is significant at $10 \%, 5 \%$, and $1 \%$ levels.

That is to say, the effect of social networks on sports behavior not only is influenced by the strength and density of social networks but also influenced the interaction of different social networks.

\subsubsection{Mediating Effect of Social Efficacy and the Moderating} Effect of Self-Presentation. Social efficacy plays a mediating role in the relationship between social networking and adolescent sports behavior, and these results can be explained using the following perspectives.

First, according to the theoretical model of "two paths and three layers" of trust behavior, social relationship is a situational factor, and its influence on adolescent sports behavior is affected by internal cognition [27]. In a positive and extroverted social relationship, adolescent sports behaviors tend to receive more social support, which then can enable adolescents to form a more positive internal cognition of themselves and others, thereby producing a stronger motivation to undertake sports [28].

Secondly, from the perspective of self-efficacy theory, situational condition is one of the important factors that can affect individual self-efficacy [29]. If one is in a familiar and pleasant environment, individual's self-efficacy will increase. Doing sports is an important way to relieve adolescent psychological stress and improve physical health. Thus, adolescents receive positive demonstrations and responses from their social network and their motivation to exercise will strengthen in order to achieve the recognition of their social relationships.

The moderating effect of self-presentation in the relationship between a social network and adolescent sports behavior is not significant in that the positive effect of social network on adolescent sports behavior is not affected by adolescents' self-presentation. This outcome may be because, on the one hand, the level of self-presentation in Chinese adolescents' sports is low. According to the Research Report on Internet Users' Information Security released by the China Internet Network Information Center (CNNIC, 2018), more than $73 \%$ of adolescents' self-presentation focuses on entertainment and study, not so much on sports [30]. On the other hand, although self-presentation can reduce the loneliness of individuals and show them the enthusiasm of sports behaviors, it also presents certain negative information about sports, such as fatigue and spending time, thereby weakening the positive effect of self-presentation [31].

To sum up then, this study concludes that social networking not only directly predicts adolescent sports behavior but also indirectly affects their actual sports behavior through social efficacy. The implication of this mediator model is that adolescent sports behavior can also be generated through interpersonal interaction and that behavior can be affected by individual cognition, thereby showing the relevance of self-cognition gained by social interaction [32].

\section{Conclusions}

The main conclusions of this study are the following:

(1) The social networks of adolescents have obvious positive effects on their sports behavior. Single online social networks and offline social networks, instrumental networks, emotional networks, and mixed networks have obvious positive effects on adolescent sports behavior, respectively. However, under the influence of multiple types of social networks, offline 
social networking has a negative predictive effect on adolescent sports behavior, while the effect of mixed networks is not so obvious.

(2) Social efficacy plays an intermediary role in the relationship between social networking and adolescent sports behavior. The moderating effect of self-presentation is not significant.

Although this paper found the importance of social networks in adolescents sports behavior and discovered the role of social efficacy, there are some limitations to this study, which need to be addressed in future studies. For example, all variables in this study were investigated using a questionnaire; it can measure variables in static level but may ignore diversity and complexity of variables; furthermore, this study found that the moderating effect of self-presentation is not significant. This finding may have a theoretical rationale, but it may also be due to the low degree of self-presentation of the sample. In the future, that sample needs to be expanded for further research and more precise evaluation.

\section{Data Availability}

The survey data used to support the findings of this study are available from the corresponding author upon request.

\section{Conflicts of Interest}

The authors declare that there are no conflicts of interest regarding the publication of this paper.

\section{Acknowledgments}

This study was supported by the MOE (Ministry of Education in China) Project of Humanities and Social Sciences (Project no. 18YJC890012).

\section{References}

[1] L. P. Spear, "The adolescent brain and age-related behavioral manifestations," Neuroscience \& Biobehavioral Reviews, vol. 24, no. 4, pp. 417-463, 2000.

[2] L. Steinberg and K. C. Monahan, "Age differences in resistance to peer influence," Developmental Psychology, vol. 43, no. 6, pp. 1531-1543, 2009.

[3] S. Sarah-Jeanne, N. R. James, C. B. Julie et al., "Effect of peers and friends on youth physical activity and motivation to be physically active," Journal of Pediatric Psychology, vol. 34, no. 2, pp. 217-225, 2009.

[4] M. Jennifer, D. L. H. Kayla, L. M. Barnett et al., "Friendship network characteristics are associated with physical activity and sedentary behavior in early adolescence," PLOS ONE, vol. 10, no. 12, pp. 1-15, 2015.

[5] M. J. Edwards, R. Jago, J. S. Simon et al., "The influence of friends and siblings on the physical activity and screen viewing behaviours of children aged 5-6 years: a qualitative analysis of parent interviews," BMJ Open, vol. 5, no. 5, Article ID e006593, 2015.

[6] K. D. L. Haye, G. Robins, P. Mohr et al., "How physical activity shapes, and is shaped by, adolescent friendships," Social Science \& Medicine, vol. 73, no. 5, pp. 719-728, 2011.
[7] Z. Li, F. Xiong, X. Wang et al., "Topological influence-aware recommendation on social networks," Complexity, vol. 2019, Article ID 6325654, 12 pages, 2019.

[8] B. Yu and X. Hu, "Toward training and assessing reproducible data analysis in data science education," Data Intelligence, vol. 1, no. 4, pp. 333-344, 2019.

[9] A. Bandura, "Self-efficacy mechanism in human agency," American Psychologist, vol. 37, no. 2, pp. 122-147, 1982.

[10] A. Bandura, Self-efficacy: The Exercise of Control, Worth Publishers, New Nork, NY, USA, 1997.

[11] C. Fan and A. S. Mak, "Measuring social self-efficacy in a culturally diverse student population," Social Behavior and Personality: An International Journal, vol. 26, no. 2, pp. 131144, 1998

[12] M. Wei, D. W. Russell, and R. A. Zakalik, "Adult attachment, social self-efficacy, self-disclosure, loneliness, and subsequent depression for freshman college students: a longitudinal study," Journal of Counseling Psychology, vol. 52, no. 4, pp. 602-614, 2005.

[13] F. Xiong, W. Shen, H. Chen et al., "Exploiting implicit influence from information propagation for social recommendation," IEEE Transactions on Cybernetics, 2019.

[14] H. Y. Mao, H. C. Hsu, and S. D. Lee, "Gender differences in related influential factors of regular exercise behavior among people in Taiwan in 2007: a cross-sectional study," PLoS one, vol. 15, no. 1, pp. 1-22, 2020.

[15] S. Park, J. Y. Kang, and L. A. Chadiha, "Social network types, health, and health-Care use among South Korean older adults," Research on Aging, vol. 40, no. 2, pp. 131-154, 2018.

[16] E. J. Jeong and D. H. Kim, "Social activities, self-efficacy, game attitudes, and game addiction," Cyberpsychology, Behavior, and Social Networking, vol. 14, no. 4, pp. 213-221, 2011.

[17] J. Kim and J.-E. R. Lee, "The Facebook paths to happiness: effects of the number of Facebook friends and self-presentation on subjective well-being," Cyberpsychology, Behavior, and Social Networking, vol. 14, no. 6, pp. 359-364, 2011.

[18] Z. Wen and B. Ye, "Analyses of mediating effects: the development of methods and models," Advances in Psychological Science, vol. 22, no. 5, pp. 731-745, 2014.

[19] L. G. Smith, L. Banting, R. Eime et al., "The association between social support and physical activity in older adults : a systematic review," International Journal of Behavioral Nutrition \& Physical Activity, vol. 14, no. 1, p. 56, 2017.

[20] K. P. Smith and N. A. Christakis, "Social networks and health," Annual Review of Sociology, vol. 34, no. 1, pp. 405-429, 2008.

[21] S. Aral and C. Nicolaides, "Exercise contagion in a global social network," Nature Communications, vol. 8, Article ID 14753, 2017.

[22] S. Ji, S. Pan, E. Cambria et al., "A survey on knowledge graphs: representation, acquisition and applications," 2020, http:// arxiv.org/abs/2002.00388.

[23] T. W. Valente, K. Fujimoto, C.-P. Chou, and D. Spruijt-Metz, "Adolescent affiliations and adiposity: a social network analysis of friendships and obesity," Journal of Adolescent Health, vol. 45, no. 2, pp. 202-204, 2009.

[24] P. Manasatchakun, P. Chotiga, J. Hochwälder, Å. Roxberg, M. Sandborgh, and M. Asp, "Factors associated with healthy aging among older persons in northeastern Thailand," Journal of Cross-Cultural Gerontology, vol. 31, no. 4, pp. 369-384, 2016.

[25] L. Adam and P. S. Damon, "The intersection of sport management and sociology of sport research: a social network perspective," Sport Management Review, vol. 15, no. 2, pp. 244-256, 2012. 
[26] Z. Wu, S. Pan, F. Chen et al., "A comprehensive survey on graph neural networks," IEEE Transactions on Neural Networks and Learning Systems, vol. 99, 2020.

[27] L. N. Howard, "Sport sociology, NASSS, and undergraduate education in the United States: a social network perspective for developing the field," Sociology of Sport Journal, vol. 27, no. 1, pp. 76-88, 2010.

[28] Q. Zhu, "Self-disclosure in online support groups for people living with depression," Master's Thesis, National University of Singapore, Singapore, 2011.

[29] F. Xiong, X. Wang, S. Pan, H. Yang, H. Wang, and C. Zhang, "Social recommendation with evolutionary opinion dynamics," IEEE Transactions on Systems, Man, and Cybernetics: Systems, 2019.

[30] L. S. Eller, E. L. Lev, C. Yuan, and A. V. Watkins, "Describing self-care self-efficacy: definition, measurement, outcomes, and Implications : definition,measurement, outcomes, and implications," International Journal of Nursing Knowledge, vol. 29, no. 1, pp. 38-48, 2018.

[31] B. Cornwell, L. P. Schumm, E. O. Laumann et al., "Assessment of social network change in a national longitudinal survey," Journals of Gerontology, vol. 69, no. 2, pp. 75-82, 2014.

[32] L. Guo, Q. Zhang, W. Hu, Z. Sun, and Y. Qu, "Learning to complete knowledge graphs with deep sequential models," Data Intelligence, vol. 1, no. 3, pp. 224-243, 2019. 